\title{
Baixo consumo de frutas, verduras e legumes: fatores associados em idosos em capital no Centro-Oeste do Brasil
}

\author{
Low consumption of fruit, vegetables and greens: \\ associated factors among the elderly in a Midwest Brazilian city
}

Erika Aparecida Silveira ${ }^{1}$

Bruna Bittar Martins ${ }^{2}$

Laísa Ribeiro Silva de Abreu ${ }^{3}$

Camila Kellen de Souza Cardoso ${ }^{1}$
${ }^{1}$ Programa de PósGraduação em Ciências da Saúde, Faculdade de Medicina, Universidade Federal de Goiás. R. 236 s/n, Setor Leste Universitário. 74605-050 Goiânia GO Brasil.erikasil@terra.com.br

${ }^{2}$ Universidade Gama Filho.

${ }^{3}$ Faculdade de Saúde

Pública, USP.

\begin{abstract}
The scope of the study was to evaluate the prevalence of daily consumption of fruit, vegetables and greens by the elderly and its association with sociodemographic, lifestyle, morbidity and hospitalization variables. The study was part of the multiple-stage sampling cross-sectional research entitled the Goiannia Elderly Project (Projeto Idosos Goiânia). 416 elderly people were interviewed in their homes. Multivariate analysis was conducted using Poisson regression to analyze statistical associations. $P$ values of $<.05$ were considered statistically significant. Daily consumption of fruit, vegetables and greens was 16.6\%: fruit accounted for $44 \%$, vegetables $39.7 \%$ and greens $32.5 \%$. Factors statistically associated with daily consumption of fruits and vegetables were female sex, age between 70 and 79, higher education lev$e l$, social class $A / B$ and $C$, alcohol consumption, use of sweeteners, regular physical activity during leisure time, abdominal obesity and hospitalization. Public policies to promote health should develop strategies that encourage adequate intake of fruit, vegetables and greens among the elderly, since regular consumption of same can improve quality of life and prevent/control diseases.
\end{abstract}

Key words Elderly, Food intake, Chronic disease, Physical activity, Abdominal obesity
Resumo O objetivo foi avaliar a prevalência do consumo diário de frutas, verduras e legumes (FVL) em idosos e sua associação com fatores sociodemográficos, estilo de vida, presença de morbidades e hospitalização. Esta pesquisa faz parte do Projeto Idosos Goiânia, estudo transversal com amostragem em múltiplos estágios. Foram entrevistados 416 idosos em seus domicílios. Realizouse análise multivariada por Regressão de Poisson para investigar os fatores associados ao consumo de FVL ao nivel de significância de 5\%. A prevalência de consumo diário de FVL foi de 16,6\%, sendo de frutas $44 \%$, verduras $39,7 \%$ e legumes $32,5 \%$. O consumo diário de FVL foi associado a: sexo feminino, idade entre 70 e 79 anos, maior escolaridade, classe social A/B e C, consumo de bebida alcoólica, uso de adoçantes, prática regular de atividade fisica no lazer, obesidade abdominal e hospitalização. É importante desenvolver estratégias de promoção da alimentação saudável com ênfase na ingestão adequada de FVL na população idosa, tendo em vista seu potencial na prevenção e controle de doenças.

Palavras-chave Idoso, Consumo de alimentos, Doença crônica, Atividade física, Obesidade abdominal 


\section{Introdução}

Observa-se um aumento da longevidade mundial nas últimas décadas. A população de pessoas com 65 anos ou mais no mundo dobrou de 7\% para 14\% no Reino Unido, nos Estados Unidos e na França ${ }^{1}$. As estimativas apontam que nas próximas quatro décadas a população idosa atinja $22 \%$ da população mundial ${ }^{2}$. No Brasil, o percentual de idosos passou de 9,1\% em 1999 para $11,3 \%$ em 20093. Desse modo, o envelhecimento populacional torna-se um desafio no campo da Saúde Pública, constituindo um cenário inédito no qual o número de pessoas desta faixa etária ultrapassará o de menores de cinco anos ${ }^{4}$. Assim manter a saúde nessa faixa etária é um aspecto importante para a qualidade de vida dos idosos, para os gestores dos serviços de saúde e para a sociedade.

A alimentação saudável tem um importante papel na manutenção da saúde, com destaque para o consumo de frutas, verduras e legumes (FVL) que trazem benefícios e seu consumo inadequado apresenta-se como um dos dez principais fatores de risco para a carga global de doenças $^{1,5}$. A presença de FVL na dieta favorece o suprimento de micronutrientes, fibras e outros componentes com propriedades funcionais, protege o DNA contra danos e, juntamente com a atividade física regular, contribui para a regulação da gordura corporal nos idosos, sendo esses fatores determinantes do desenvolvimento das doenças crônicas não transmissíveis (DCNTs) ${ }^{6-10}$. Investigações prospectivas em países europeus verificaram que o alto consumo de FVL reduz em $11 \%$ o risco de morte quando comparado com o baixo $^{11}$.

Estudos realizados nos Estados Unidos mostraram associação do consumo adequado de FVL ou de dieta rica em fibras, incluindo FVL, com menor incidência de doenças cardíacas em ido$\operatorname{sos}^{12,13}$, bem como câncer ${ }^{14}$, diabetes tipo $2^{15}$, doenças periodontais ${ }^{16-18}$, menor risco de inflamação e melhor função renal ${ }^{19}$. O consumo adequado de frutas está associado também a um melhor nível de massa óssea ${ }^{20}$.

Conforme evidências, o consumo adequado de frutas, verduras e legumes durante a vida adulta é associado a menor probabilidade de doenças crônicas 5 . Contudo, é importante investigar a associação entre a presença de doenças crônicas como diabetes, hipertensão arterial e obesidade e o consumo diário de FVL em idosos. Essa é uma das abordagens a que este estudo se propõe, tendo em vista sua relevância para o controle das doenças crônicas em idosos e, ainda, considerando que as poucas pesquisas com estes, que avaliaram o consumo de FVL, verificaram a associação apenas com aspectos sociodemográfi$\cos ^{21-23}$.

Os determinantes de consumo de FVL em idosos são bastante complexos e envolvem muito além das questões socioeconômicas e demográfi$\mathrm{cas}^{5}$. Sendo assim, é importante aprofundar o conhecimento sobre esses aspectos. Portanto, o objetivo do presente estudo foi avaliar a prevalência do consumo diário de frutas, legumes e verduras e sua associação com fatores sociodemograficos, estilo de vida, presença de morbidades e hospitalização em idosos não institucionalizados.

\section{Métodos}

O presente estudo faz parte de um estudo maior intitulado "Situação de saúde e indicadores antropométricos para avaliação do estado nutricional de idosos usuários do Sistema Único de Saúde de Goiânia-GO", mais conhecido como "Projeto Idosos Goiânia". Os dados apresentados no presente estudo compõem uma amostra de idosos $\geq 60$ anos de idade, usuários das unidades básicas de saúde/SUS e residentes em Goiânia-GO. Empregou-se os seguintes critérios de exclusão: idosos incapazes de deambular, com deficiência mental ou dificuldade cognitiva e/ou auditiva para responder as questões no momento da entrevista.

A amostragem foi realizada em múltiplos estágios, primeiramente por sorteio aleatório a partir do arquivo ativo das unidades saúde dentre aqueles que procuraram atendimento nos doze meses anteriores a pesquisa. O município era dividido em nove distritos sanitários (DS) e o endereço dos idosos foi localizado na base de dados do Instituto Brasileiro de Geografia e Estatística (IBGE).

Maiores detalhes sobre todas as etapas dos procedimentos de amostra e amostragem podem ser consultados em outras publicações do Projeto Idosos ${ }^{24-26}$. A coleta de dados foi realizada por duplas compostas por um entrevistador e um antropometrista, que efetuaram visita domiciliar aos idosos entre os meses de maio de 2008 e fevereiro de 2009.

Quanto à avaliação antropométrica, as duplas foram previamente capacitadas e padronizadas para realizá-la, conforme protocolo de Habicht ${ }^{27}$. Foi coletado o peso, a altura e a circunferência da cintura. Destaca-se que, as medidas foram 
realizadas conforme as técnicas propostas por Lohman et al..$^{28}$.

A aferição do peso foi procedida com balança eletrônica da marca Tanita (UM080W), tipo digital portátil e com capacidade para $200 \mathrm{~kg}$ e precisão de $100 \mathrm{~g}$. A altura foi obtida utilizando fita métrica com precisão de $0,1 \mathrm{~cm}$, fixada em parede sem rodapé, com auxílio de fio de prumo e esquadro de madeira. A partir dessas medidas chegou-se ao Índice de Massa Corporal (IMC). $\mathrm{O}$ estado nutricional foi categorizado de acordo com Lipschtz ${ }^{29}$, considerando sobrepeso/obesidade aqueles com IMC $>27 \mathrm{~kg} / \mathrm{m}^{2}$, os quais são discutidos por Silveira et al..$^{30}$ para uso em idosos brasileiros.

A circunferência da cintura foi determinada com uma fita métrica colocada no ponto médio entre a porção inferior da última costela e a crista ilíaca, do lado direito do corpo, de modo a não pressionar. Utilizou a classificação segundo risco de complicações metabólicas associadas à obesidade abdominal, proposta pela OMS ${ }^{31}$, sendo o ponto de corte de $88 \mathrm{~cm}$ para mulheres e $102 \mathrm{~cm}$ para homens.

Foram coletados dados socioeconômicos e demográficos, como idade, estado civil, escolaridade em anos de estudos e classe social (A, B, C e E), sendo esta determinada segundo os critérios da Associação Brasileira das Empresas de Pesquisas $^{32}$, além de números de pessoas que vivem no domicílio e situação de trabalho. Quanto às informações sobre estilo de vida, coletou-se dados de tabagismo, consumo de bebida alcoólica, uso diário de adoçantes e prática de atividade no lazer segundo o International Physical Activity Questionnaire (IPAQ) ${ }^{33}$. As variáveis sobre as condições de saúde foram: hospitalização no último ano, obesidade, obesidade abdominal, hipertensão arterial e diabetes mellitus. Essas últimas por meio da pergunta: "Quais doenças o médico já disse que o $\operatorname{Sr}(a)$ têm?". Era marcado o que o idoso referia sem ler nenhuma opção de resposta $^{34}$.

Para avaliação do consumo de frutas, verduras e legumes (FVL) foram realizadas as seguintes perguntas: 1) em geral, com que frequência o Sr. (a) come fruta?; 2) em geral, com que frequência $o$ Sr. (a) bebe suco de fruta fresca?; 3) em geral, com que frequência o Sr. (a) come verduras (vegetais folhosos)? e 4) em geral, com que frequência o Sr. (a) come legumes (cenoura, vagem, abobrinha) frescos/ cozidos?. Antes de iniciar essa serie de perguntas o idoso era instruído para pensar no seu hábito alimentar costumeiro nos últimos doze meses. Em seguida à pergunta, lia-se as opções de resposta: a) nunca; b) menos que uma vez por mês; c) uma vez ao mês; d) 2 a 3 vezes por mês; e) 1 a 2 vezes por semana;f) 3 a 4 vezes por semana; g) 5 ou 6 vezes por semana; h) uma vez ao $\mathrm{dia}^{35}$. No manual de instruções dos entrevistadores possuía exemplos de verduras (alface, rúcula, agrião, couve) e legumes (cenoura, beterraba, chuchu, abóbora, vagem, couve-flor) e os mesmos foram treinados para a aplicação do questionário de forma a padronizar a coleta dessas informações.

O desfecho (sim/não) foi o consumo diário de frutas e/ou suco de frutas mais verduras e legumes. Assim, o idoso deveria comer esses três tipos de alimentos diariamente. $\mathrm{O}$ consumo diário mínimo de FVL foi baseado na recomendação da World Health Organization (WHO) de 400 gramas/dia ${ }^{36}$, porém aplicou-se como variável desfecho o consumo desses três alimentos pelo menos uma vez ao dia.

O banco de dados foi construído no software Epi Data 3.1 em dupla entrada para posterior análise de consistência interna da digitação. Para a análise estatística foi utilizado o software estatístico STATA 12.0.

Os resultados foram expressos em frequências absoluta e relativa. Calculou-se a prevalência do consumo diário de FVL para as variáveis estudadas. A associação do consumo diário de FVL com as demais variáveis foi estimada por meio da Regressão de Poisson simples, utilizando-se como medida de efeito a Razão de Prevalência (RP) com intervalos de confiança de 95\% (IC95\%).

Posteriormente, as variáveis que apresentaram na análise bivariada valor $\mathrm{p}<0,20$ foram incluídas no modelo de análise multivariada hierarquizada por Regressão de Poisson. Para tanto, as variáveis foram ordenadas em $1^{\circ}$ nível: sociodemográficas, $2^{\circ}$ nível: estilo de vida; $3^{\circ}$ nível: condições de saúde. Permaneceram no modelo final somente as variáveis com significância de $5 \%$.

Este estudo foi aprovado pelo Comitê de Ética em Pesquisa da Universidade Federal de Goiás e a entrevista com o idoso foi autorizada mediante assinatura do termo de consentimento livre e esclarecido (TCLE). Essa pesquisa adotou os princípios estabelecidos na Declaração de Helsinque da Associação Médica Mundial.

\section{Resultados}

Foram investigados 416 idosos e a prevalência de consumo diário de frutas e verduras foi de $16,6 \%$. 
Avaliando somente o consumo de frutas a prevalência foi de $44 \%$. O consumo de frutas mais verduras e verduras mais legumes foi de $24 \%$ e o consumo desses dois tipos de alimentos esteve significativamente associado $(\mathrm{p}=0,000)$ (Figura 1 e Tabela 1).

As características da amostra de idosos estudada podem ser observadas na Tabela 2, em que se destaca que $65,9 \%$ eram do sexo feminino, $48,5 \%$ entre 60 e 69 anos, $50,2 \%$ da classe social C e $33,6 \%$ realizavam atividade física no lazer. As variáveis sociodemográficas e estilo de vida foram significativamente associadas ao consumo de FVL: sexo, idade, classe social, anos de estudo, tabagismo, atividade física no lazer e uso de adoçante (Tabela 2).

Com relação às condições de saúde, $27,2 \%$ eram obesos, $51,7 \%$ hipertensos, $76,4 \%$ com obesidade abdominal e $24,5 \%$ foram hospitalizados. Entre as variáveis sobre as condições de saúde,

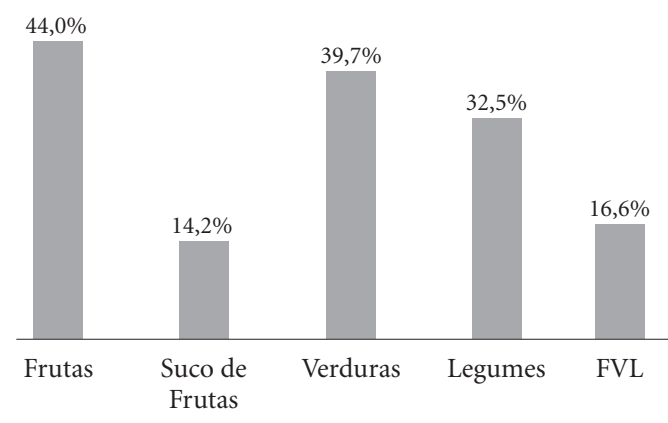

Figura 1. Prevalência do consumo diário de frutas, suco de frutas, verduras e legumes em idosos. Projeto Idosos/Goiânia ( $\mathrm{n}=416)$.

Tabela 1. Prevalência do consumo diário de dois tipos de frutas, suco de frutas, verduras e legumes e teste de associação. Projeto Idosos/Goiânia, $(\mathrm{n}=416)$.

\begin{tabular}{lrrr}
\hline \multicolumn{1}{c}{ Consumo diário } & \multicolumn{1}{c}{$\mathbf{n}$} & \multicolumn{1}{c}{$\%$} & Valor $^{*}$ \\
\hline Fruta + suco de fruta & 32 & 7,69 & 0,087 \\
Fruta + verduras & 102 & 24,52 & 0,000 \\
Fruta + legumes & 85 & 20,43 & 0,000 \\
Verduras + legumes & 100 & 24,04 & 0,000 \\
FVL (fruta + suco de frutas & 69 & 16,60 & \\
+ verduras + legumes) & & & \\
\hline
\end{tabular}

*Teste qui-quadrado de Pearson. foram associados ao consumo de FVL: obesidade, obesidade abdominal e hipertensão arterial. A hospitalização ficou no limiar da significância com valor $=\mathrm{p}=0,053$ (Tabela 3 ).

Após a análise bivariada foram incluídos na análise multivariada por regressão de Poisson as seguintes variáveis: sexo, idade, classe social, anos de estudo, numero de pessoas no domicilio, trabalho, tabagismo, bebida alcoólica, atividade física no lazer, uso de adoçante, obesidade, obesidade abdominal, hipertensão arterial e hospitalização. Mantiveram-se associadas significativamente ao consumo diário de FVL após analise multivariada: sexo feminino, idade entre 70-79, $\geq 9$ anos de estudo, classe social A/B e C, realizar atividade física no lazer, consumir bebida alcóolica, usar adoçante, ter obesidade abdominal e ter sido hospitalizado (Tabela 4).

\section{Discussão}

Trata-se de um dos primeiros estudos a avaliar o consumo de frutas, verduras e legumes em idosos fora da região sudeste do Brasil ${ }^{22}$, assim apresenta um panorama de outra região do país e com cenário socioeconômico diferente. Outro aspecto que vale enfatizar como contribuição da presente pesquisa é a investigação sobre a presença de algumas doenças crônicas, hospitalização e atividade física, pois não foram localizados estudos que avaliaram essas associações com o consumo de FVL.

No presente estudo, menos de um quinto $(16,7 \%)$ da população idosa consumia diariamente FVL. Embora exista grande oferta e diversidade de frutas e hortaliças no Brasil ${ }^{37}$, nota-se uma baixa ingestão desses alimentos, muito aquém das recomendações da $\mathrm{OMS}^{36}$. Viebig et al..$^{22}$, observaram cerca de $35,0 \%$ dos idosos de baixa renda em São Paulo sem consumo diário de nenhum tipo de fruta ou hortaliça. Pesquisa realizada no Irã, encontrou um panorama melhor, visto que a prevalência do consumo diário de cinco ou mais porções de frutas e hortaliças foi de $37,9 \%$ entre idosos ${ }^{38}$.

Já pesquisa em adultos, entre 20 e 65 anos, em uma cidade do sul do Brasil, somente $20,9 \%$ dos entrevistados relatou consumo regular de frutas, legumes e verduras ${ }^{39}$. Em geral, estudos demonstraram que menos de $10 \%$ da população brasileira atinge as recomendações de consumo de $\mathrm{FVL}^{3,21,40}$. Logo, observa-se que a ingestão ainda está muito abaixo do priorizado, seja no consumo isolado ou associado de FVL, inclusive entre os idosos. 
Tabela 2. Distribuição da amostra, prevalência, razão de prevalência (RP) e intervalo de confiança de 95\% (IC 95\%) do consumo diário de frutas, verduras e legumes de acordo com variáveis sociodemográficas e estilo de vida de idosos. Goiânia-GO, $(n=416)$.

\begin{tabular}{|c|c|c|c|c|c|}
\hline \multirow{2}{*}{ Variável } & \multicolumn{2}{|c|}{$\begin{array}{c}\text { Distribuição da } \\
\text { amostra }(n=416) \\
\end{array}$} & \multirow{2}{*}{$\begin{array}{l}\text { Prevalência de } \\
\text { consumo diário } \\
\text { de FVL n }(\%)\end{array}$} & \multirow{2}{*}{ RP (IC 95\%) } & \multirow{2}{*}{ Valor $\mathrm{p}^{*}$} \\
\hline & $\mathbf{n}$ & $\%$ & & & \\
\hline Sexo & & & & & 0,000 \\
\hline Feminino & 274 & 65,87 & $53(19,34)$ & $1,72(1,24-2,37)$ & \\
\hline Masculino & 142 & 34,13 & $16(11,27)$ & 1,00 & \\
\hline Idade em anos & & & & & 0,009 \\
\hline $60-69$ & 203 & 48,53 & $32(15,76)$ & $1,81(0,99-3,30)$ & \\
\hline $70-79$ & 168 & 40,19 & $33(19,76)$ & $2,27(1,24-4,14)$ & \\
\hline$\geq 80$ & 47 & 11,24 & $4(8,51)$ & 1,00 & \\
\hline Estado civil & & & & & 0,680 \\
\hline Casado & 229 & 54,78 & $37(16,16)$ & 1,00 & \\
\hline Solteiro, viúvo ou separado & 187 & 45,22 & $32(17,11)$ & $1,06(0,81-1,39)$ & \\
\hline Classe social & & & & & 0,000 \\
\hline $\mathrm{A}$ e B & 42 & 10,1 & $12(28,57)$ & $2,62(1,72-3,99)$ & \\
\hline $\mathrm{C}$ & 209 & 50,24 & $39(18,66)$ & $1,71(1,24-2,36)$ & \\
\hline $\mathrm{D}$ e $\mathrm{E}$ & 165 & 39,66 & $18(10,91)$ & 1,00 & \\
\hline Anos de estudo & & & & & 0,001 \\
\hline $0-4$ & 265 & 71,12 & $37(13,96)$ & 1,00 & \\
\hline $5-8$ & 72 & 19,25 & $13(18,06)$ & $1,29(0,90-1,86)$ & \\
\hline$\geq 9$ & 36 & 9,63 & $11(30,56)$ & $2,19(1,48-3,23)$ & \\
\hline No pessoas por domicílio & & & & & 0,068 \\
\hline Sozinho & 40 & 9,62 & $7(17,50)$ & 1,00 & \\
\hline 1 & 140 & 33,65 & $28(20,00)$ & $1,14(0,71-1,84)$ & \\
\hline 4 & 204 & 49,04 & $31(15,20)$ & $0,87(0,54-1,39)$ & \\
\hline 5 & 32 & 7,69 & $3(9,38)$ & $0,53(0,24-1,17)$ & \\
\hline Trabalho & & & & & 0,198 \\
\hline Sim & 61 & 14,66 & $8(13,11)$ & $0,76(0,50-1,17)$ & \\
\hline Não & 355 & 85,33 & $61(17,18)$ & 1,00 & \\
\hline Tabagismo & & & & & 0,035 \\
\hline $\operatorname{Sim}$ & 41 & 9,86 & $4(9,76)$ & 1,00 & \\
\hline Não & 375 & 90,14 & $65(17,33)$ & $1,78(0,99-3,18)$ & \\
\hline Bebida alcoólica & & & & & 0,150 \\
\hline Sim & 63 & 15,14 & $13(20,63)$ & $1,30(0,92-1,84)$ & \\
\hline Não & 353 & 84,86 & $56(15,86)$ & 1,00 & \\
\hline Atividade física no lazer & & & & & 0,000 \\
\hline Sim & 140 & 33,65 & $32(22,86)$ & $1,70(1,30-2,24)$ & \\
\hline Não & 276 & 66,35 & $37(13,41)$ & 1,00 & \\
\hline Uso de adoçante & & & & & 0,014 \\
\hline Sim & 118 & 28,37 & $25(21,19)$ & $1,43(1,08-1,90)$ & \\
\hline Não & 298 & 71,63 & $44(14,77)$ & 1 & \\
\hline
\end{tabular}

${ }^{*}$ Teste de Wald.

No presente estudo, encontrou-se uma prevalência de consumo diário de frutas ( $44 \%)$, suco de frutas $(14,2 \%)$, verduras $(39,7 \%)$ e legumes $(32,5 \%)$, ou seja, todos inferiores a $50 \%{ }^{41}$. Neutzling et al. ${ }^{39}$ evidenciaram também que menos da metade dos adultos consumiam regularmente frutas $(43,5 \%)$, legumes $(36,4 \%)$ e verduras $(41,5 \%)$. Essa condição se mostra preocupante, pois, sabe-se que o risco de desenvolvimento de câncer colorretal em pessoas entre 50 e 71 anos de idade é reduzido em 20 a $30 \%$ quando o consumo de FVL é diário ${ }^{42}$. Estudo de coorte verificou que a adesão às recomendações da OMS ${ }^{36}$, para ingestão de FVL, possui efeito real na proteção contra o câncer de bexiga, pois as mulheres que consumiam diariamente FVL apresentaram 
Tabela 3. Distribuição da amostra, prevalência, razão de prevalência (RP) e intervalo de confiança de 95\% (IC 95\%) do consumo diário de frutas, verduras e legumes de acordo com variáveis sobre condição de saúde de idosos. Goiânia, Goiás, $(\mathrm{n}=416)$.

\begin{tabular}{|c|c|c|c|c|c|}
\hline \multirow{2}{*}{ Variável } & \multicolumn{2}{|c|}{$\begin{array}{c}\text { Distribuição da } \\
\text { amostra }(n=416)\end{array}$} & \multirow{2}{*}{$\begin{array}{l}\text { Prevalência de } \\
\text { consumo diário } \\
\text { de FVL n }(\%)\end{array}$} & \multirow{2}{*}{ RP (IC 95\%) } & \multirow{2}{*}{ Valor $\mathrm{p}$} \\
\hline & $\mathbf{n}$ & $\%$ & & & \\
\hline Obesidade & & & & & 0,000 \\
\hline Sim & 113 & 27,16 & $27(23,89)$ & $1,72(1,30-2,28)$ & \\
\hline Não & 303 & 72,84 & $42(13,86)$ & 1,00 & \\
\hline Obesidade abdominal & & & & & 0,006 \\
\hline Sim & 316 & 76,33 & $58(18,35)$ & $1,63(1,13-2,37)$ & \\
\hline Não & 98 & 23,67 & $11(11,22)$ & 1,00 & \\
\hline Hipertensão arterial & & & & & 0,019 \\
\hline Sim & 208 & 51,74 & $38(18,27)$ & $1,41(1,05-1,89)$ & \\
\hline Não & 194 & 48,26 & $30(15,46)$ & 1,00 & \\
\hline \multicolumn{6}{|l|}{ Diabetes Mellitus } \\
\hline Sim & 98 & 23,61 & $17(17,35)$ & $1,06(0,77-1,45)$ & 0,730 \\
\hline Não & 317 & 76,38 & $52(16,40)$ & 1,00 & \\
\hline Hospitalização & & & & & 0,053 \\
\hline Sim & 102 & 24,52 & $21(20,59)$ & $1,35(1,00-1,81)$ & \\
\hline Não & 314 & 75,48 & $48(15,29)$ & 1,00 & \\
\hline
\end{tabular}

*Teste de Wald.

$65 \%$ menos risco de ter essa neoplasia, quando comparadas com aquelas que não alcançavam a recomendação ${ }^{42}$. Os Estados Unidos desenvolvem esforços para aumentar esse consumo por meio de políticas públicas, como o Programa Alimentar Five a day e as novas recomendações da MyPyramid. O primeiro passo foi aumentar a recomendação diária de ingestão de FVL devido aos altos índices de obesidade e incidência de DCNTs naquele país ${ }^{43-45}$.

Trabalhos realizados com adultos em cidades de outras regiões do Brasil assemelham-se aos achados deste estudo, apresentando consumo de frutas, verduras e legumes substancialmente superior entre as mulheres e os mais ve$\operatorname{lhos}^{21,39,40,46,47}$. Outros países também têm evidenciado essa mesma tendência de maior consumo de vegetais no sexo feminino, como comprovado nos estudos com a população adulta da Finlândia, Estônia, Lituânia e Látvia, República Checa, Hungria, Eslováquia, Eslovênia, Espanha, Suazilândia, Ucrânia, Uruguai e Vietna ${ }^{48,49}$. Provavelmente, esses achados estão implicados na maior preocupação por parte das mulheres e das pessoas mais velhas com uma alimentação mais saudável. Alguns estudos relatam que crenças e motivação para controle de peso poderiam explicar as diferenças no comportamento alimentar entre os sexos, sendo importante para as mulheres na es- colha do alimento questões relacionadas à saúde, prazer, conveniência e preço $0^{50,51}$.

$\mathrm{O}$ aumento da escolaridade foi associado significativamente com o consumo de FVL, resultados semelhantes a outros estudos ${ }^{47,52}$. O baixo consumo de frutas, verduras e legumes foi estatisticamente associado a pouca escolaridade, a baixa renda familiar e a não inserção no mercado de trabalho em outras pesquisas ${ }^{53,54}$. Em adultos de Portugal, o consumo de frutas e vegetais aumentava significativamente com os anos de estudo, em ambos os $\operatorname{sexos}^{55}$. Estudo com adultos de 23 centros europeus constatou maior consumo de frutas e vegetais associados ao sexo feminino e a maior formação educacional ${ }^{14}$.

Destaca-se também na presente pesquisa, a associação de classe social com o consumo de frutas, verduras e legumes, com aumento do consumo nas classes mais altas. Este resultado corrobora ao estudo de Neutzling et al. ${ }^{39}$, em adultos no sul do Brasil. Bowman ${ }^{56}$ afirma que idosos com menor renda consomem em menor frequência e quantidade FVL em comparação aqueles com maior poder aquisitivo. No estudo World Health Survey (WHS), conduzido pela World Health Organization em outros 33 países, verificou-se que consumo de frutas e vegetais era diretamente proporcional à renda ${ }^{49}$. Assim, pode-se ressaltar que o maior nível de informação, 
Tabela 4. Regressão de Poisson múltipla por níveis hierárquicos para variáveis associadas ao consumo diário de frutas, verduras e legumes em idosos. Goiânia, Goiás, $(n=416)$.

\begin{tabular}{|c|c|c|c|}
\hline Variáveis & Razão de Prevalência ajustada & IC $95 \%$ & Valor $\mathbf{p}$ \\
\hline \multicolumn{4}{|l|}{$1^{\circ}$ nível } \\
\hline \multicolumn{4}{|l|}{ Sexo } \\
\hline Masculino & 1,00 & & \\
\hline Feminino & 1,96 & $1,40-2,75$ & 0,000 \\
\hline \multicolumn{4}{|l|}{ Idade (anos) } \\
\hline $60-69$ & 1,18 & $0,64-2,18$ & 0,602 \\
\hline $70-79$ & 1,84 & $1,00-3,38$ & 0,047 \\
\hline$\geq 80$ & 1,00 & & \\
\hline \multicolumn{4}{|l|}{ Anos de estudo } \\
\hline $0-4$ & 1,00 & & \\
\hline $5-8$ & 1,23 & $0,85-1,79$ & 0,276 \\
\hline$\geq 9$ & 1,94 & $1,23-3,05$ & 0,004 \\
\hline \multicolumn{4}{|l|}{ Classe social } \\
\hline $\mathrm{A}$ e B & 2,46 & $1,48-4,08$ & 0,000 \\
\hline $\mathrm{C}$ & 1,89 & $1,33-2,69$ & 0,000 \\
\hline $\mathrm{D}$ e $\mathrm{E}$ & 1,00 & & \\
\hline \multicolumn{4}{|l|}{$2^{\circ}$ nível } \\
\hline \multicolumn{4}{|c|}{ Atividade física no lazer } \\
\hline Não & 1,00 & & \\
\hline Sim & 1,72 & $1,28-2,31$ & 0,000 \\
\hline \multicolumn{4}{|l|}{ Bebida alcoólica } \\
\hline Não & 1,00 & & \\
\hline Sim & 1,58 & $1,09-2,30$ & 0,016 \\
\hline \multicolumn{4}{|l|}{ Adoçantes } \\
\hline Não & 1,00 & & \\
\hline Sim & 1,38 & $1,01-1,89$ & 0,040 \\
\hline \multicolumn{4}{|l|}{ 3o nível } \\
\hline \multicolumn{4}{|c|}{ Obesidade abdominal } \\
\hline Não & 1,00 & & \\
\hline Sim & 1,60 & $1,16-2,20$ & 0,004 \\
\hline \multicolumn{4}{|l|}{ Hospitalizações } \\
\hline Não & 1,00 & & \\
\hline Sim & 1,65 & $1,20-2,26$ & 0,002 \\
\hline
\end{tabular}

${ }^{*}$ Estatística Wald.

ou seja, maior escolaridade e o melhor poder de aquisitivo são relevantes para a determinação do consumo de FVL.

Nesta pesquisa, a análise entre atividade física no lazer, uso de adoçantes dietéticos e o consumo de FVL revelou associação significativa, sendo que os indivíduos ativos e usuários de edulcorantes consomem mais FVL. Trata-se de achado relevante, ainda mais considerando que não foram encontrados estudos que analisaram essas associações em idosos. Estudo em cidade no Sul do Brasil encontrou associação do consumo de FVL com atividade física no lazer em população adulta ${ }^{39}$. Estudo sobre qualidade da dieta em adultos americanos encontrou associação entre melhor qualidade da dieta e uso frequente de adoçantes ${ }^{57}$. Acredita-se que esses indivíduos possuam uma maior preocupação com a saúde e controle de peso, logo apresentam hábitos mais saudáveis, como o regular consumo de FVL, redução de açúcar pelo uso de adoçantes e pratica de atividade física.

Outra contribuição da presente pesquisa foi a observação da associação entre o consumo diário de FVL e o uso de bebidas alcoólicas, pois não foram localizados outros estudos que realizaram essa investigação em idosos. Estudo de coorte em dez países da Europa, o EPIC - European Prospective Investigation into Cancer and Nutrition, analisando o risco de câncer e o consumo de frutas e vegetais, verificou associação entre o alto consumo e ingestão leve de bebida alcoólica (menos de 
$5 \mathrm{~g} / \mathrm{d})$. O EPIC encontrou ainda que o alto consumo de frutas e vegetais protege (0.90 IC95\% 0.96 - 0.94) de câncer especificamente relacionados ao consumo de bebida alcoólica (trato digestivo alto, mama, fígado e colorectum) em análise de bebedores pesados, ou seja, acima de $60 \mathrm{~g} / \mathrm{d}$ em homens e $30 \mathrm{~g} / \mathrm{d}$ em mulheres ${ }^{14}$. Em adultos americanos foi observada associação inversa entre consumo elevado de bebida alcoólica e ingestão de frutas e vegetais entre aqueles de menor renda, porém sem associação entre os de maiores níveis de rendimentos ${ }^{58}$. O presente estudo, não avaliou a quantidade de bebidas alcoólicas em gramas/ dia para verificar se o consumo era leve, moderado ou pesado.

Não foram localizados estudos que avaliaram a associação com estado nutricional, obesidade abdominal, diabetes e hipertensão arterial em idosos para traçar um panorama com a presente pesquisa. Entre as morbidades estudas a obesidade abdominal manteve-se associada ao consumo diário de FVL, com risco de 1,6 vezes (razão de prevalência ajustada). Em adultos maiores de 35 anos, houve associação entre obesidade abdominal e consumo adequado de FVL, porém com efeito protetor $(\text { Odds Ratio }=0.77)^{59}$. Esse mesmo estudo não observou associação entre pressão arterial e glicemia de jejum ${ }^{59}$, sendo similar à presente pesquisa. Estudo com adultos verificou que não houve associação entre estado nutricional e consumo de FVL, também análogo aos achados desta pesquisa ${ }^{39}$. O EPIC avaliou os quartis de consumo de frutas e vegetais conforme o IMC e não observou diferença entre os estra$\operatorname{tos}^{11,60}$.

$\mathrm{Na}$ Espanha, estudo transversal com 4.393 pessoas com padrão de dieta do mediterrâneo, com alto consumo de gorduras, encontrou que a associação entre elevado consumo de frutas e vegetais foi inversamente associado aos níveis de pressão arterial $^{61}$, porém não há como realizar um paralelo com a presente pesquisa devido ao diferente padrão alimentar e a faixa etária.

Ainda sobre a presença de morbidades, não foi observada associação com diabetes e o consumo de FLV, diferente do observado em coorte de adultos americanos entre 25 e 74 anos, no entanto não foram encontradas analises somente com indivíduos idosos ${ }^{61}$.
Da mesma forma que a presença de doenças crônicas, a associação com hospitalização encontrada na presente pesquisa, não foi avaliada em outras sobre consumo de $\mathrm{FVL}^{11,14,22,39,40,59}$. Essa associação com hospitalizações pode ser um exemplo de causalidade reversa, sendo que após a hospitalização os idosos foram recomendados por profissionais de saúde para melhorar a alimentação com a inclusão de FLV.

Apesar das limitações de um estudo transversal e as peculiaridades de se estudar consumo alimentar, os dados demonstraram validade externa, considerando a concordância com estudos realizados no Brasil e em diversos países.

Sabe-se que, a ingestão desses alimentos é recomendada por instituições internacionais e integra políticas de saúde pública relevantes ${ }^{36}$. A meta-análise reforça ainda mais a importância do consumo diário desses alimentos, mostrando diminuição da taxa de mortalidade por todas as causas, especialmente de mortes por doenças de alta prevalência em idosos, como as de origem cardiovascular e o câncer ${ }^{13}$.

Portanto, conclui-se que o consumo de frutas, verduras e legumes na população idosa estudada foi baixa e corrobora com outras pesquisas nacionais e internacionais. Os achados do presente estudo apontam que o consumo diário de frutas, verduras e legumes foi muito baixo e que os fatores associados foram: sexo feminino, idade entre 70 e 79 anos, maior escolaridade e classe social A, B e C, consumo de bebida alcoólica, uso de adoçantes dietéticos, prática regular de atividade física no lazer, obesidade abdominal e hospitalização. Essas informações colaboram para aumentar o conhecimento sobre o tema e ampliar a discussão para investigações de outros fatores associados ao consumo de FVL, até então não analisados. Considerando que o consumo regular desses alimentos é capaz de auxiliar na prevenção de diversas doenças crônicas, reduzir a mortalidade e melhorar a qualidade de vida dos idosos, recomenda-se o desenvolvimento de políticas públicas e programas de promoção da saúde com estratégias que estimulem a ingestão adequada de FVL, especialmente entre os idosos com menor consumo, ou seja, os homens, sedentários, de menor renda e menor escolaridade. 


\section{Colaboradores}

EA Silveira, coordenadora do Projeto Idosos/ Goiânia em que o estudo apresentado está inserido, participou da coordenação da coleta de dados; da concepção e do planejamento; da análise e da interpretação dos dados; da redação e da revisão crítica do conteúdo; e, da aprovação da versão final do manuscrito. BB Martins e LRS Abreu participaram da coleta de dados; do planejamento do artigo; da análise estatística e da interpretação dos dados; da redação do texto; e, da aprovação da versão final. CKS Cardoso participou da revisão de literatura, planejamento do artigo, interpretação dos dados, redação do texto, formatação e aprovação da versão final.

\section{Referências}

1. Prince MJ, Wu F, Guo Y, Robledo LMG, O’Donnell $\mathrm{M}$, Sullivan R, Yusuf S. The burden of disease in older people and implications for health policy and practice. Lancet 2015; 385(9967):549-562.

2. Bloom DE, Chatterji S, Kowal P, Lloyd-Sherlock P, McKee M, Rechel B, Rosenberg L, Smith JP. Macroeconomic implications of population ageing and selected policy responses. Lancet 2015; 385(9968):649-657.

3. Instituto Brasileiro de Geografia e Estatística (IBGE). Coordenação de Trabalho e Rendimento. Pesquisa de orçamentos familiares 2008-2009: análise do consumo alimentar pessoal no Brasil. Rio de Janeiro: IBGE; 2011.

4. Suzman R, Beard JR, Boerma T, Chatterji S. Health in an ageing world - what do we know? Lancet 2015; 385(9967):484-486.

5. Nicklett EJ, Kadell AR. Fruit and vegetable intake among older adults: a scoping review. Maturitas 2013; 75(4):305-312.

6. Rolls BJ, Ello-Martin JA, Tohill BC. What can intervention studies tell us about the relationship between fruit and vegetables consumption and weight management? Nutr Rev 2004; 62(1):1-17.

7. Vilaça KHC, Ferriolli E, Lima NKC, De Paula FJA, Marchini JS, Moriguti JC. Força muscular e densidade mineral óssea em idosos eutróficos e desnutridos. Rev Nutr 2011; 24(6):845-852.

8. Carneiro G, Faria NA, Ribeiro Filho FF, Guimarães A, Lerário D, Ferreira SRG, Zanella MT. Influência da distribuição da gordura corporal sobre a prevalência de hipertensão arterial e outros fatores de risco cardiovascular em indivíduos obesos. Rev Assoc Med Bras 2003; 49(3):306-311.

9. Santos RD, Gagliardi ACM, Xavier HT, Magnoni CD, Cassani R, Lottenberg AMP, Casella Filho A, Araújo DB, Cesena FY, Alves RJ, Fenelon G, Nishioka SAD, Faludi AA, Geloneze B, Scherr C, Kovacs C, Tomazzela C, Carla C, Barrera-Arellano D, Cintra D, Quintão E, Nakandakare ER, Fonseca FAH, Pimentel I, Santos JE, Bertolami MC, Rogero M, Izar MC, Nakasato M, Damasceno NRT, Maranhão R, Cassani RSL, Perim R, Ramos S. I Diretriz sobre consumo de gorduras e doenças cardiovasculares. Arq Bras Cardiol 2013; 100(1 Supl 3):1-40.

10. Sardinha AN, Canella DS, Martins APB, Claro RM, Levy RB. Dietary sources of fiber intake in Brazil. Appetite 2014; 79:134-138.

11. Leenders M, Sluijs I, Ros MM, Boshuizen HC, Siersema PD, Ferrari P, Weikert C, Tjønneland A, Olsen A, Boutron-Ruault MC, Clavel-Chapelon F, Nailler L,Teucher B, Li K, Boeing H, Bergmann MM, Trichopoulou A, Lagiou P, Trichopoulos D, Palli D, Pala V, Panico S, Tumino R, Sacerdote C, Peeters PH, van Gils CH, Lund E, Engeset D, Redondo ML, Agudo A, Sánchez MJ, Navarro C, Ardanaz E, Sonestedt E, Ericson U, Nilsson LM, Khaw KT, Wareham NJ, Key TJ,Crowe FL, Romieu I, Gunter MJ, Gallo V, Overvad K, Riboli E, Bueno-deMesquita HB. Fruit and vegetable consumption and mortality: European prospective investigation into cancer e nutrition. Am J Epidemiol 2013; 178(4):590602. 
12. Hartley L, Igbinedion E, Holmes J, Flowers N, Thorogood M, Clarke A, Stranges S, Hooper L, Rees K. Increased consumption of fruit and vegetables for the primary prevention of cardiovascular diseases. Cochrane Database Syst Rev 2013; 6:1-39.

13. Wang X, Ouyang Y, Liu J, Zhu M, Zhao G, Bao W, Hu FB. Fruit and vegetable consumption and mortality from all causes, cardiovascular disease and cancer: systematic review and dose-response meta-analysis of prospective cohort studies. BMJ 2014; 349:1-14.

14. Boffeta P, Couto E, Wichmann J, Ferrari P, Trichopoulos D, Bueno-de-Mesquita A HB, van Duijnhoven FJ, Büchner FL, Key T, Boeing H, Nöthlings U, Linseisen J, Gonzalez CA, Overvad K, Nielsen MR, Tjønneland A, Olsen A, Clavel-Chapelon F, Boutron-Ruault MC, Morois S, Lagiou P, Naska A, Benetou V, Kaaks R, Rohrmann S, Panico S, Sieri S, Vineis P, Palli D, van Gils CH, Peeters PH, Lund E, Brustad M, Engeset D, Huerta JM, Rodríguez L, Sánchez MJ, Dorronsoro M, Barricarte A, Hallmans G, Johansson I, Manjer J, Sonestedt E, Allen NE, Bingham S, Khaw KT, Slimani N, Jenab M, Mouw T, Norat T, Riboli E, Trichopoulou A. Fruit and vegetable intake and overall cancer risk in the European prospective investigations into cancer e nutrition (EPIC). $J$ Natl Cancer Inst 2010; 102(8):529-537.

15. Cooper AJ, Forouhi NG, Ye Z, Buijsse B, Arriola L, Balkau, Barricarte A, Beulens JW, Boeing H, Büchner FL, Dahm CC, de Lauzon-Guillain B, Fagherazzi G, Franks PW, Gonzalez C, Grioni S, Kaaks R, Key TJ, Masala G, Navarro C, Nilsson P, Overvad K, Panico S, Ramón Quirós J, Rolandsson O, Roswall N, Sacerdote C, Sánchez MJ, Slimani N, Sluijs I, Spijkerman AM, Teucher B, Tjonneland A, Tumino R, Sharp SJ, Langenberg C, Feskens EJ, Riboli E, Wareham NJ; InterAct Consortium. Fruit and vegetable intake and type 2 diabetes: EPIC-InterAct prospective study and meta-analysis. Eur J Clin Nutr 2012; 66(10):1082-1092.

16. Nishida M, Grossi SG, Dunford RG, Ho AW, Trevisan $\mathrm{M}$, Genco RJ. Dietary vitamin C and the risk for periodontal disease. J Periodontol 2000; 71(8):1215-1223.

17. Giskes K, Turrel G, Patterson C, Newman B. Socioeconomic differences among Australian adults in consumption of fruit and vegetables and intake of vitamins A, C and folate. J Hum Nutr Dietet 2002; 15(5):375-385.

18. Alagl AS, Bhat SG. Ascorbic acid: new role of an age-old micronutrient in the management of periodontal disease in older adults. Geriatr Gerontol 2014; 15(3):241254.

19. Xu H, Huang X, Risérus U, Krishnamurthy VM, Cederholm T, Arnlöv J, Lindholm B, Sjögren P, Carrero JJ. Dietary fiber, kidney function, inflammation, and mortality risk. Clin J Am Soc Nephrol 2014; 9(12):21042110.

20. Liu Z, Leung J, Wong SY, Wong CKM, Chan R, Woo J. Greater fruit intake was associated with better bone mineral status among Chinese elderly men and women: results of Hong Kong Mr. Os and Ms. Os Studies. J Am Med Dir Assoc 2015; 16(4):309-315.
21. Jaime PC, Monteiro CA. Fruit and vegetable intake by Brazilian adults, 2003. Cad Saude Publica 2005; 21(Supl.):S19-S24.

22. Viebig RF, Pastor-Valero M, Scazufca M, Menezes PR. Consumo de frutas e hortaliças por idosos de baixa renda na cidade de São Paulo. Rev Saude Publica 2009; 43(5):806-813.

23. Riediger ND, Moghadasian MH. Patterns of fruit and vegetable consumption and the influence of sex, age and sociodemographic factors among the Canadian elderly. J Am Coll Nutr 2007; 27(2):306-313.

24. Pagotto V, Silveira EA, Velasco WD. Perfil das hospitalizações e fatores associados em idosos usuários do SUS. Cien Saude Colet 2013; 18(10):3061-3070.

25. Silveira EA, Pagotto V, Nakatani AYK. Fatores associados à autovaliação de saúde ruim em idosos usuários do Sitema Único de Saúde. Cad Saude Publica 2011; 27(8):1593-1602.

26. Silveira EA, Dalastra L, Pagotto V. Polifarmácia, doenças crônicas e marcadores nutricionais em idosos. Rev Bras Epidemiol 2014; 17(4):818-829.

27. Habicht JP. Estandarizacion de metodos epidemiologicos cuantitativos sobre el terreno. Bol Oficina Sanit Panam 1974; 76(5):375-384.

28. Lohman TG, Roche AF, Martorel R. Anthropometric standardization reference manual. Illinois: Human Kinetics Books; 1988.

29. Lipschitz DA. Screening for nutritional status in the elderly. Prim Care 1994; 21(1):55-67.

30. Silveira EA, Gilberto KAC, Barbosa LS. Prevalência e fatores associados à obesidade em idosos residentes em Pelotas, Rio Grande do Sul, Brasil: classificação da obesidade segundo dois pontos de corte do índice de massa corporal. Cad Saude Publica 2009; 25(7):1569-1577.

31. World Health Organization (WHO). Obesity. Preventing and managing the global epidemic. Geneva: Report of a WHO Consultation on Obesity; 1997.

32. Associação Brasileira das Empresas de Pesquisas (Abep). Critério de Classificação Econômica do Brasil. São Paulo: Abep; 2008.

33. Benedetti TR, Bertoldo PCA, Ciro RRA, Giovana ZM, Édio LP. Reprodutibilidade e validade do Questionário Internacional de Atividade Física (IPAQ) em homens idosos. Rev Bras Med Esporte 2007; 13(1):11-16.

34. Malta DC, Iser BPM, Claro RM, Moura L, Bernal RTI, Nascimento AF, Silva Júnior JB, Monteiro CA. Prevalência de fatores de risco e proteção para doenças crônicas não transmissíveis em adultos: estudo transversal, Brasil, 2011. Epidemiol Serv Saúde 2013; 22(3):423-434.

35. Gibson RS. Measuring food consumption of individuals. In: Gibson RS. Principles of nutritional assessment. New York: Oxford University Press; 2005.

36. World Health Organization (WHO). Fruit and vegetable promotion initiative - report of the meeting. Geneva: WHO; 2003. 
37. Levy RB, Claro RM, Mondini L, Sichieri R, Monteiro CA. Distribuição regional e socioeconômica da disponibilidade domiciliar de alimentos no Brasil em 20082009. Rev Saude Publica 2012; 46(1):6-15.

38. Sabzghabaee AM, Mirmoghtadaee P, Mohammadi M. Fruit and vegetable consumption among community dwelling elderly in an iranian population. Int J Prev Med 2010; 1(2):98-102.

39. Neutzling MB, Rombaldi AJ, Azevedo MR, Hallal PC. Fatores associados ao consumo de frutas, legumes e verduras em adultos de uma cidade no Sul do Brasil. Cad Saude Publica 2009; 25(11):2365-2374.

40. Assumpção D, Domene SMA, Fisberg RM, Barros MBA. Qualidade da dieta e fatores associados entre idosos: estudo de base populacional em Campinas, São Paulo, Brasil. Cad Saude Publica 2014; 30(8):16801694.

41. Park Y, Subar AF, Kipinis V, Thompson FE, Mouw T, Hollenbeck A, Leitzmann MF, Schatzkin A. Fruit and vegetable intakes and risk of colorectal cancer in the NIH-AARP diet and health study. Am J of Epidemiol 2007; 166(2):170-180.

42. Park SY, Ollberding NJ, Woolcott CG, Wilkens LR, Henderson BE, Kolonel LN. Fruit and vegetable intakes are associated with lower risk of bladder cancer among women in the multiethnic Cohort Study. J Nutr 2013; 143(8) $1283-1292$

43. United States of America (US). Centers for Disease Control and Prevention. 5 A Day Works! Atlanta: US Department of Health and Human Services; 2005.

44. United States of America (US). Department of Agriculture and Department of Health and Human Services. Dietary guidelines for americans. $7^{\text {th }}$ ed. Washington: US Government Printing Office; 2010.

45. Ungar N, Sieverding M, Stadnitski T. Increasing fruit and vegetable intake: "Five a day" versus "just one more". Appetite 2013; 65:200-204.

46. Campos VC, Bastos JL, Gauche H, Boing AF, Assis MAA. Fatores associados ao consumo adequado de frutas, legumes e verduras em adultos de Florianópolis. Rev Bras Epidemiol 2010; 13(2):352-362.

47. Figueiredo ICR, Jaime FPC, Monteiro CA. Fatores associados ao consumo de frutas, legumes e verduras em adultos da cidade de São Paulo. Rev Saude Publica 2008; 42(5):777-785.

48. Prättälä R, Paalanen L, Grinberga D, Helasoja V, Kasmel A, Petkeviciene J. Gender differences in the consumption of meat, fruit and vegetable are similar in Finland and the Baltic countries. Eur J Public Health 2006; 17(5):520-525.

49. Hall JN, Moore S, Harper SB, Lynch JW. Global variability in fruit and vegetable consumption. Am J Prev Med 2009; 36(5):402-409.

50. Westenhoefer J. Age and gender dependente profile of food choice. Forum Nutr 2005; (57):44-51.
51. Konttinen H, Sarlio-Lähteenkorva S, Silventoinen K, Männistö S, Haukkala A. Socio-economic disparities in the consumption of vegetables, fruit and energy-dense foods: the role of motive priorities. Public Health Nutr 2013; 16(5):873-882.

52. Jaime PC, Figueiredo ICR, Moura EC, Malta DC. Fatores associados ao consumo de frutas e hortaliças no Brasil, 2006. Rev Saude Publica 2009; 43(S2):57-64.

53. Moghadasian MH. Patterns of fruit and vegetable consumption and the influence of sex, age and sociodemographic factors among the Canadian elderly. $\mathrm{J} \mathrm{Am} \mathrm{Coll}$ Nutr 2007; 27(2):306-313.

54. Jorge MIE, Martins IS, Araújo EAC. Diferenciais socioeconômicos e comportamentais no consumo de hortaliças e frutas em mulheres residentes em município da região metropolitana de São Paulo. Rev Nutr 2008; 21(6):695-703.

55. Moreira PA, Padrão PD. Educational and economic determinants of food intake in Portuguese adults: a cross-sectional survey. BMC Public Health 2004; 4(58):1-11.

56. Bowman S. Low economic status is associated with suboptimal intakes of nutritious foods by adults in the National Health and Nutrition Examination Survey 1999-2002. Nutr Res 2007; 27(9):515-523.

57. Drewnowski A, Rehm CD. Consumption of low-calorie sweeteners among US adults is associated with higher healthy eating index (HEI 2005) scores and more physical activity. Nutrients 2014; 6(10):4389-4403.

58. Shimotsu ST, Jones-Webb RJ, Lytle LA, MacLehose RF, Nelson TF, Forster JL. The relationships among socioeconomic status, fruit and vegetable intake, and alcohol consumption. Am J Health Promot 2012; 27(1):21-28.

59. Castanho GKF, Marsola F, Mclellan KCP, Nicola M, Moreto F, Burini RC. Consumo de frutas, verduras e legumes associado à síndrome metabólica e seus componentes em amostra populacional adulta. Cien Saude Colet 2013; 18(2):385-392.

60. Alonso A, de la Fuente C, Martín-Arnau Am, de Irala J, Martínez JA, Martínez-González MA. Fruit and vegetable consumption is inversely associated with blood pressure in a Mediterranean population with a high vegetable-fat intake: the Seguimiento Universidad de Navarra (SUN) Study. Br J Nutr 2004; 92(2):311-319.

61. Ford ES, Mokdad AH. Fruit and vegetable consumption and diabetes mellitus incidence among US adults. Prev Med 2001; 32(1):33-39.

Artigo apresentado em 04/03/2015

Aprovado em 07/07/2015

Versão final apresentada em 09/07/2015 
